
allemande

48-2 | 2016

Les espaces publics des pays germanophones, des espaces publics transnationaux?

\title{
La transposition du régime matrimonial de droit allemand dans le cadre du divorce en France
} Chronique juridique

\section{Sandie Calme}

\section{OpenEdition Journals}

Édition électronique

URL : https://journals.openedition.org/allemagne/421

DOI : 10.4000/allemagne.421

ISSN : 2605-7913

Éditeur

Société d'études allemandes

\section{Édition imprimée}

Date de publication : 28 décembre 2016

Pagination : 497-504

ISSN : 0035-0974

Référence électronique

Sandie Calme, "La transposition du régime matrimonial de droit allemand dans le cadre du divorce en France ", Revue d'Allemagne et des pays de langue allemande [En ligne], 48-2 | 2016, mis en ligne le 28 décembre 2016, consulté le 18 mai 2021. URL : http://journals.openedition.org/allemagne/421 ; DOI https://doi.org/10.4000/allemagne.421 


\section{Chronique juridique}

\section{La transposition du régime matrimonial de droit allemand dans le cadre du divorce en France}

\section{- Sandie Calme*}

Le règlement 2201/2003 du 27 novembre 2003 relatif à la compétence, la reconnaissance et l'exécution des décisions en matière matrimoniale et en matière de responsabilité parentale abrogeant le règlement (CE) n 1347/2000 s'applique, dans les situations présentant un conflit de lois, au divorce et à la séparation de corps. Dans le cadre des procédures de divorce en France présentant un élément d'extranéité, il offre une pluralité d'alternatives pour ce qui est du choix de l'État membre où sera introduite la procédure de divorce, ce qui autorise de façon récurrente le choix de l'assignation en divorce auprès des juridictions françaises, avec un système de compétence juridictionnelle exclusive dès lors que l'époux assigné en divorce a sa résidence habituelle sur le territoire d'un État membre ou est ressortissant d'un État membre ou, dans le cas du Royaume-Uni et de l'Irlande, a son «domicile» sur le territoire de l'un de ces États membres. On retrouve une grande flexibilité avec le règlement (CE) $n^{\circ} 4 / 2009$ du Conseil du 18 décembre 2008 relatif à la compétence, la loi applicable, la reconnaissance et l'exécution des décisions et la coopération en matière d'obligations alimentaires, pour ce qui est de la compétence juridictionnelle concernant les obligations alimentaires relatives au divorce.

Le règlement $\mathrm{n}^{\circ}$ 1259/2010 du Conseil du 20 décembre 2010 mettant en œuvre une coopération renforcée dans le domaine de la loi applicable au divorce et à la séparation de corps s'applique au divorce, sauf pour ce qui est, notamment, des effets patrimoniaux du mariage, de la responsabilité parentale et obligations alimentaires, selon son article premier. Son article 8 établit la loi applicable au divorce autour des critères de résidence habituelle des parents, de nationalité commune des époux et de compétence de la juridiction saisie: dans son champ d'application, la loi applicable au divorce peut

* Docteur en droit, LL.M. (Francfort-sur-le-Main, Allemagne), avocate au barreau de Paris. 
toujours être celle de la juridiction saisie, entre autres alternatives du même rang. Selon le Protocole de La Haye du 23 novembre 2007 sur la loi applicable aux obligations alimentaires, qui vaut pour la France et pour l'Allemagne, la loi de la résidence habituelle du créancier est amenée à s'appliquer concernant les obligations alimentaires, sauf pour des raisons d'ordre public, et en prenant en considération les ressources du débiteur. La Convention du 19 octobre 1996 concernant la compétence, la loi applicable, la reconnaissance, l'exécution et la coopération en matière de responsabilité parentale et de mesures de protection des enfants dispose que la responsabilité parentale, et également la question de son retrait, relèvent de la loi de la résidence habituelle de l'enfant.

La question de la nationalité du régime matrimonial dans le cadre du divorce semble être la seule à échapper à ce degré de flexibilité. En effet, traditionnellement, le régime matrimonial est lié à un droit national et relève des spécificités locales. En présence d'éléments d'extranéité, il apparaît nécessaire de définir le régime juridique du régime matrimonial des époux dans le cadre de la procédure de divorce. Afin d'y parvenir, dans le cadre du divorce en France où le régime matrimonial est à la base de droit allemand, la Convention de La Haye du 14 mars 1978 sur la loi applicable aux régimes matrimoniaux (I) est porteuse de solutions. Elle est en harmonie avec la jurisprudence française régissant les mariages formés antérieurement à son entrée en vigueur et est reprise en substance par une proposition de nouveau règlement européen. Quant au droit allemand du régime matrimonial, il est relativement encadré d'interdits (II). Comme il prohibe le choix d'un régime matrimonial étranger, l'accord franco-allemand instituant un régime matrimonial optionnel de la participation aux acquêts, également ouvert aux autres États membres de l'Union européenne, apporte au droit allemand un ultième régime matrimonial, tout en concrétisant le concept de régime matrimonial multinational (III).

\section{L'implication de la Convention de La Haye du 14 mars 1978 sur la loi applicable aux régimes matrimoniaux}

En droit international privé français, pour ce qui est de déterminer la loi applicable aux régimes matrimoniaux, on distingue les situations extérieures au cadre de la Convention de La Haye du 14 mars 1978 (A) de celles qui lui sont inhérentes (B). En tout état de cause, l'esprit de la Convention de La Haye est préservé, également dans le cadre de la proposition (C) de Règlement européen du Conseil relatif à la compétence, la loi applicable, la reconnaissance et l'exécution des décisions en matière de régimes matrimoniaux.

\section{A. Cas où la Convention de La Haye ne s'applique pas}

Pour les mariages formés préalablement au $1^{\text {er }}$ septembre 1992, date d'entrée en vigueur de cette convention de 1978, les solutions apportées sont surtout jurisprudentielles. Il s'agit de prendre en compte un faisceau d'indices, tels que la volonté des parties lors du mariage, des circonstances postérieures au mariage, le premier domicile matrimonial, le lieu où est localisé le centre des intérêts personnels et matériels des époux. Ceci donne lieu à une certaine marge d'appréciation.

\section{B. Cas d'application de la Convention de La Haye}

Conformément à son article 2, la Convention de La Haye du 14 mars 1978 revêt un caractère universel: elle s'applique à tous les mariages célébrés après le $1^{\text {er }}$ septembre 
1992, même si la nationalité, la résidence habituelle des époux ou la loi applicable en vertu de cette convention ne sont pas celles d'un État contractant, ce qui est confirmé par la jurisprudence de la Cour de cassation (Civ. $1^{\text {re }}, 12$ novembre 2009, pourvoi $\mathrm{n}^{\circ}$ 08-18343, Bulletin 2009, I, n 224). Observons toutefois qu'il s'agissait d'un cas où la Cour de cassation concluait à l'application du régime matrimonial légal de droit français et qu'il n'y a pas de jurisprudence fournie concluant à l'application d'un régime matrimonial étranger.

Elle est en vigueur en droit français, luxembourgeois et néerlandais.

Elle ne régit pas les obligations alimentaires entre époux, les droits de succession de l'époux survivant ni la capacité des époux (article $1^{\mathrm{er}}$ ).

Le principe est celui du choix (articles 3 et 6) par les époux de la loi applicable à leur union: "Le régime matrimonial est soumis à la loi interne désignée par les époux avant le mariage» ou "au cours du mariage», mais ils ne peuvent désigner que «la loi d'un État dont l'un des époux a la nationalité au moment de cette désignation », "la loi de l'État sur le territoire duquel l'un des époux a sa résidence habituelle au moment de cette désignation» ou «la loi du premier État sur le territoire duquel l'un des époux établira une nouvelle résidence habituelle après le mariage», cette loi s'appliquant à la totalité de leurs biens, à cela près qu'ils "peuvent désigner, en ce qui concerne les immeubles ou certains d'entre eux, la loi du lieu où ces immeubles sont situés» voire "prévoir que les immeubles qui seront acquis par la suite seront soumis à la loi du lieu de leur situation». Préalablement au mariage, il est possible d'opter pour «la loi du premier État sur le territoire duquel l'un des époux établira une nouvelle résidence habituelle après le mariage».

Selon l'article 4 de la Convention de La Haye sur la loi applicable aux régimes matrimoniaux du 14 mars 1978, en l'absence de nationalité commune et à défaut de choix de loi, le régime matrimonial des époux est soumis à la loi interne de l'État de leur première résidence habituelle après le mariage.

Toutefois, le régime matrimonial est soumis à la loi interne de l'État de la nationalité commune des époux (article 4 in fine):

- dès lors que cette nationalité est celle des Pays-Bas, ou,

- si l'État de nationalité commune des époux n'est pas Partie à la présente convention, dès lors que sa loi interne est applicable selon son droit international privé, et que les époux établissent leur première résidence habituelle après le mariage aux Pays-Bas ou dans un État qui n'est pas Partie à la Convention et dont le droit international privé prescrit également l'application de leur loi nationale,

- dès lors que les époux n’ont pas établi sur le territoire du même État leur première résidence habituelle après le mariage.

En l'absence de résidence habituelle des époux sur le territoire du même État et de nationalité commune, le régime matrimonial sera soumis à la loi interne de l'État avec lequel, compte tenu de toutes les circonstances, il présente les liens les plus étroits.

La dérogation propre aux Pays-Bas n'aura pas d'effet pour des époux qui conservent tous deux leur résidence habituelle sur le territoire d'un État autre que les Pays-Bas où, au moment du mariage, l'un et l'autre avaient leur résidence habituelle depuis cinq ans au moins, ou sur le territoire d'un État non Partie à la Convention et dont le droit international privé prescrit l'application de la loi nationale (article 5 alinéa 2). 
Par principe, la loi applicable selon la présente Convention s'applique tant que les époux n'en ont désigné aucune autre, et ce même en cas de changement de nationalité ou de résidence habituelle (article 7 alinéa ${ }^{\mathrm{er}}$ ).

À défaut de choix (article 7 in fine), la loi interne de l'État où ils ont tous deux leur résidence habituelle devient applicable, en lieu et place de celle à laquelle leur régime matrimonial était antérieurement soumis :

- dès lors qu'ils y fixent leur résidence habituelle, si la nationalité de cet État est ou devient leur nationalité commune, ou

- lorsque, après le mariage, cette résidence habituelle a duré plus de dix ans, ou

- dès lors qu'ils y fixent leur résidence habituelle, si le régime matrimonial est soumis à la loi interne de l'État de la nationalité commune des époux au seul motif que les époux n’avaient pas établi sur le territoire du même État leur première résidence habituelle après le mariage.

Ce changement de la loi applicable n'a d'effet que pour l'avenir (article 8), les biens appartenant aux époux antérieurement à ce changement n'étant en principe pas soumis à la loi désormais applicable, sauf choix des parties selon des formes prescrites pour le contrat de mariage.

Des dispositions spéciales régissent l'opposabilité aux tiers du régime matrimonial.

Les conditions relatives au consentement des époux quant à la loi déclarée applicable sont déterminées par cette loi (article 10).

La désignation de la loi applicable doit faire l'objet d'une stipulation expresse ou résulter indubitablement des dispositions d'un contrat de mariage (article 11).

Le contrat de mariage, valable quant à la forme si celle-ci répond soit à la loi interne applicable au régime matrimonial, soit à la loi interne en vigueur au lieu où le contrat a été passé, doit toujours faire l'objet d'un écrit daté et signé des deux époux (article 12).

La désignation par stipulation expresse de la loi applicable doit revêtir la forme prescrite pour les contrats de mariage, soit par la loi interne désignée, soit par la loi interne du lieu où intervient cette désignation et elle doit toujours faire l'objet d'un écrit daté et signé des deux époux (article 13).

Des considérations d'ordre public peuvent évincer la loi désignée par la présente Convention, en cas d'incompatibilité manifeste avec l'ordre public (article 14). Elle n'est donc pas immuable.

\section{Perspectives d'avenir de la Convention de La Haye}

Avec la proposition de Règlement européen du Conseil relatif à la compétence, la loi applicable, la reconnaissance et l'exécution des décisions en matière de régimes matrimoniaux, la Convention est susceptible d'être remplacée par un nouveau texte, à une certaine échelle.

Cette proposition prévoit l'unité de la loi applicable au régime matrimonial, couvrant l'ensemble des biens des époux (article 15), ainsi que l'universalité (article 21) de la règle de conflit de lois, la loi applicable pouvant ne pas être celle d'un État membre du règlement proposé. Elle s'inscrit dans la continuité de la Convention de La Haye.

Dans ce cadre, le choix de loi applicable au régime matrimonial, de la part des époux, pourra porter (article 16):

- sur la loi de l'État de la résidence habituelle commune des époux ou futurs époux, ou 
- sur la loi de l'État de la résidence habituelle d'un des époux ou futurs époux au moment de ce choix, ou

- sur la loi d'un État dont l'un des époux ou futurs époux possède la nationalité au moment de ce choix.

À défaut de choix par les époux, la loi applicable au régime matrimonial (article 17) sera:

- la loi de l'État de la première résidence habituelle commune des époux après le mariage, ou à défaut,

- la loi de l'État de la nationalité commune des époux au moment du mariage, sauf s'ils ont plus d'une nationalité commune, ou à défaut,

- la loi de l'État avec lequel les époux ont ensemble les liens les plus étroits, compte tenu de toutes les circonstances, et notamment du lieu de célébration du mariage.

Les époux pourront, à tout moment au cours du mariage, soumettre leur régime matrimonial à une loi autre que celle jusqu'alors applicable (article 18), mais ne pourront désigner que l'une des lois suivantes:

- la loi de l'État de la résidence habituelle d'un des époux au moment de ce choix, - la loi d'un État dont l'un des époux possède la nationalité au moment de ce choix.

Si les époux choisissent expressément de donner à ce changement de loi applicable un effet rétroactif, le changement ne valant en principe que pour l'avenir, cette rétroactivité ne portera pas atteinte à la validité des actes antérieurs conclus sous l'empire de la loi jusque-là applicable, ni aux droits des tiers résultant de la loi antérieurement applicable (article 18 in fine).

Le choix de loi sera formalisé (article 19) selon la forme prescrite pour le contrat de mariage, soit par la loi applicable de l'État choisi, soit par la loi de l'État du lieu de rédaction de l'acte: il devra être au moins exprès, et formulé par un acte écrit, daté et signé par les deux époux, et si la loi de l'État membre dans lequel les deux époux ont leur résidence habituelle commune au moment du choix prévoit pour le contrat de mariage des conditions de forme supplémentaires, ces conditions devront être respectées.

La forme (article 20) du contrat de mariage sera celle prescrite soit par la loi applicable au régime matrimonial, soit par la loi de l'État du lieu de rédaction du contrat. Ce contrat devra faire au moins l'objet d'un écrit, daté et signé par les deux époux, et si la loi de l'État membre dans lequel les deux époux ont leur résidence habituelle commune au moment de la conclusion du contrat de mariage prévoit, pour ce contrat, des conditions de forme supplémentaires, ces conditions devront être respectées.

Les lois de police (article 22) et l'ordre public du for (article 23) auront toutefois priorité.

Le renvoi sera exclu (article 25).

\section{Le régime matrimonial de droit allemand}

Les régimes matrimoniaux de droit allemand sont strictement encadrés, faisant l'objet de prescriptions impératives qui affectent les procédures de divorce présentant certains éléments d'extranéité. Cela vaut tant pour le régime matrimonial légal (A) que pour les régimes matrimoniaux conventionnels (B), où la liberté contractuelle se heurte à certains interdits. 


\section{A. Le régime légal}

Selon l'article $\$ 1363$ du Code civil allemand (Bürgerliches Gesetzbuch, BGB), à défaut de contrat de mariage, les époux relèvent du régime matrimonial de la communauté d'acquêts (Zugewinngemeinschaft) (article $\$ 1363$ alinéa $1^{\text {er }}$ du Code civil allemand). Ce régime matrimonial est donc le régime matrimonial légal allemand, celui qui s'applique à défaut de contrat de mariage. Sous ce régime matrimonial, le patrimoine de chacun des époux ne constitue pas leur patrimoine commun, y compris pour ce qui est du patrimoine qu'un conjoint acquiert après la conclusion du mariage (article $\$ 1363$ alinéa 2 du Code civil allemand) : les patrimoines sont séparés pendant le mariage. L'acquêt (Zugewinn) est le montant dont est augmenté le patrimoine final d'un époux par rapport à son patrimoine de départ (article $\$ 1373$ du Code civil allemand). Cet acquêt (Zugewinn) que les époux obtiennent dans le mariage fait l'objet d’une compensation (article $\$ 1363$ alinéa 2 du Code civil allemand) en cas de divorce. Ainsi, selon l'article $\$ 1378$ alinéa $1^{\text {er }}$ du Code civil allemand, si l'acquêt d’un conjoint excède celui de l'autre, la moitié de l'excès revient à l'autre conjoint comme créance de compensation. Aussi, selon l'article $\$ 1384$ du Code civil allemand, en cas de divorce, le moment même où la requête en divorce est pendante est le point de référence pour le calcul de l'acquêt et pour l'évaluation du montant de la créance de compensation. Alors que l'article $\$ 1377$ alinéas 1 et 2 du Code civil allemand autorise les époux à procéder conjointement à l'inventaire de leur patrimoine de départ, à défaut d'inventaire du patrimoine de départ, il est présumé que le patrimoine final d'un conjoint est constitutif de son acquêt (article $\$ 1377$ alinéa 3 du Code civil allemand).

\section{B. Les régimes conventionnels}

Les époux sont en principe libres de procéder à un contrat de mariage, par acte notarié (article $\$ 1410$ ), également au cours du mariage (article $\$ 1408$ alinéa $1^{\text {er }}$ ), mais sans toutefois être autorisés à choisir un régime matrimonial qui n'existe plus ou un régime matrimonial étranger, l'article $\$ 1409$ du Code civil allemand l'interdisant expressément.

En cas de contrat de mariage stipulant la communauté des biens, des dispositions impératives encadrent la gestion des biens communs (articles 1415 et suivants du Code civil allemand).

\section{Le régime matrimonial optionnel franco-allemand}

Le constat des difficultés dans le règlement de la question de l'agencement du régime matrimonial à l'international (A), notamment dans les relations juridiques entre l'Allemagne et la France, concernant également le divorce, a justifié la mise au point de l'accord franco-allemand instituant un régime matrimonial optionnel de la participation aux acquêts (B).

\section{A. La vocation de l'accord franco-allemand}

L'accord franco-allemand instituant un régime matrimonial optionnel de la participation aux acquêts entré en vigueur le $1^{\text {er }}$ mai 2013 offre notamment aux époux binationaux ou étrangers une alternative de régime matrimonial qu'il est possible de choisir par acte de mariage notarié, avant ou pendant le mariage (article 3). 
Cet accord a une vocation transnationale: selon son article 21 alinéa $1^{\text {er }}$, "Après l'entrée en vigueur du présent accord, tout État membre de l'Union européenne peut adhérer à celui-ci. En cas d'adhésion d'un ou de plusieurs États, le Gouvernement de la République fédérale d'Allemagne agira comme dépositaire de l'accord. Les instruments d'adhésion seront déposés auprès du dépositaire.»

L'idée première est que le manque de flexibilité des dispositifs juridiques régissant les régimes matrimoniaux dans les procédures présentant des éléments d'extranéité, notamment en ce qui concerne des époux étrangers ou ayant plusieurs nationalités, rend nécessaire la mise au point d'une convention internationale instituant un régime matrimonial multinational.

\section{B. Le dispositif du régime matrimonial optionnel de la participation aux acquêts}

Dans le régime optionnel de la participation aux acquêts (article 2), le patrimoine des époux reste séparé. Les acquêts sont constitués du montant de la différence entre le patrimoine final d'un époux et son patrimoine originaire. À la dissolution du régime matrimonial, la créance de participation résulte de la comparaison des acquêts de chacun des époux.

Chaque époux a la gestion et la jouissance exclusive de son patrimoine; il en dispose seul (article 4). Néanmoins, les actes de disposition d'objets du ménage ou de droits par lesquels est assuré le logement de la famille passés par un époux sans le consentement de l'autre sont nuls, sauf s'ils sont ratifiés par l'autre époux, et un époux peut être autorisé en justice à passer seul un acte pour lequel le consentement de son conjoint serait nécessaire, si celui-ci est hors d'état de manifester sa volonté ou si son refus n'est pas justifié par l'intérêt de la famille (article 5).

En principe, chacun des époux a le pouvoir de passer seul (article 6) les contrats ayant pour objet l'entretien du ménage et l'éducation des enfants, ces contrats obligeant solidairement l'autre époux. Exceptionnellement, lorsque l'un des époux contracte des dettes dont le caractère manifestement excessif, notamment eu égard au train de vie des époux, est connu de l'autre partie au contrat ou aurait dû l'être, ces dettes n'engagent pas l'autre époux.

Des dispositions spécifiques régissent la composition du patrimoine originaire, son évaluation, la détermination du patrimoine final.

Le droit à créance de participation (article 12) s'organise comme suit:

- Si à la dissolution du régime matrimonial, les acquêts d'un époux excèdent les acquêts de l'autre époux, ce dernier peut faire valoir à l'encontre de son conjoint une créance de participation égale à la moitié de la différence.

- La créance de participation donne lieu à un paiement en argent. Toutefois, le tribunal peut, à la demande de l'un ou l'autre des époux, ordonner, à l'effet de ce paiement, le transfert de biens du débiteur au créancier, si cela répond au principe de l'équité.

- Après la dissolution du régime matrimonial, la créance de participation est transmissible à cause de mort et cessible entre vifs.

La créance de participation est déterminée en fonction de la composition et de la valeur du patrimoine des époux à la date d'introduction de la demande en divorce (article 13). 
Elle est limitée à la moitié de la valeur du patrimoine de l'époux débiteur tel qu'il existe, après déduction des dettes, à la date retenue pour la détermination du montant de cette créance (article 14).

La prescription de la créance de participation, l'obligation d'information, l'inventaire, l'octroi de délais de paiement sont également prévus.

Si la gestion de son patrimoine par l'un des époux est de nature à compromettre les droits de l'autre au titre du calcul de la créance de participation, ce dernier peut demander la liquidation anticipée de la créance de participation, la décision définitive faisant droit à la demande plaçant les époux sous le régime de la séparation de biens (article 18).

Cet accord franco-allemand reprend les grandes lignes des régimes matrimoniaux légaux français et allemand. Il se conçoit de prime abord comme un cadre à l'attention du notaire français ou allemand. Plus encore, il apporte une réponse aux difficultés concrètes de transposition du régime matrimonial légal allemand en droit français, en particulier en ce que le droit allemand de la famille s'oppose au choix d'un régime matrimonial étranger, français par exemple.

Dès lors, dans la détermination de la nationalité du régime matrimonial et du droit qui lui est applicable, pour autant que ce droit soit susceptible d'être transposé à l'étranger sur le fondement de l'équivalence fonctionnelle, il convient de rechercher l'équilibre dans le respect des considérations d'ordre public propres aux ordres juridiques concernés. Face à ces difficultés, au niveau du processus de création de normes juridiques, l'accord franco-allemand instituant un régime matrimonial optionnel de la participation aux acquêts est un précédent mettant en valeur l'idée de régime matrimonial multinational susceptible de s'étendre, au niveau de l'Union européenne et au-delà, à des situations présentant des éléments d'extranéité. Il est aussi un appel à la créativité dans la mise au point du contrat de mariage, en tant que, selon le préambule de l'accord, «régime matrimonial commun optionnel qui doit prendre place aux côtés des autres régimes matrimoniaux en vigueur dans le droit national des États contractants». 